\title{
Determinação de vitamina A no leite de mães doadoras do banco de leite humano (BLH) de Manaus/ AM: efeito do processamento
}

\author{
Ana Maria Matos Monteiro OLIVEIRA', Helyde Albuquerque MARINHO²
}

\begin{abstract}
RESUMO
Esse estudo teve como objetivo determinar os teores de vitamina A no Leite Humano Materno (LHM), de 50 mães doadoras do Banco de Leite Humano (BLH), da Maternidade Ana Braga, em Manaus-AM. As amostras foram coletadas semanalmente e mantidas congeladas até a pasteurização. As análises de retinol e $\beta$-caroteno no leite humano foram realizadas antes e após o tratamento (pasteurizaçáo), mediante o método cromatográfico. Foram retiradas alíquotas de $5 \mathrm{~mL}$ aproximadamente de leite de cada amostra. Os pesos dos bebês ao nascer das mães doadoras de leite incluídos no estudo foram $>2500 \mathrm{~g}$. A concentraçáo de vitamina A no leite tanto antes quanto após o processamento, não foram suficientes para suprir o requerimento $(46,75 \pm 6,28$ $\mu \mathrm{g} / \mathrm{dL}$ e $31,55 \pm 8,29 \mu \mathrm{g} / \mathrm{dL}$, respectivamente), de crianças que se alimentam exclusivamente do leite do BLH, notadamente as pré-termo, cujos requerimentos de vitamina A são maiores e a capacidade de ingerir leite é menor do que os nascidos a termo. A perda de vitamina A pelo processamento, em nosso estudo foi de 32,51\%. Para garantir a formação de reservas hepáticas e a proteção contra deficiência de vitamina $\mathrm{A}$, seriam necessárias concentraçôes dessa vitamina no leite superiores a $80 \mu \mathrm{g} / \mathrm{dL}$.
\end{abstract}

PalaVRAS-CHAVE: Vitamina A, Retinol, Leite Materno, Banco de Leite Humano.

\section{Determination of vitamin A in the milk of donor mothers from the human milk bank (hmb) in Manaus/AM. Effect of processing}

\section{ABSTRACT}

This study treats a transversal, descriptive, observational and analytical study with the objective of determining the Vitamin A chemical content in Human Maternal Milk (HMM) of 50 donor mothers from the Ana Braga Maternity Human Milk Bank (HMB), in Manaus, Amazonas. Samples were collected weekly and maintained frozen until pasteurization. Analyses of retinol and $\beta$-carotene in human milk were undertaken before and after the treatment (pasteurization), chromatographic method. Aliquots of approximately $5 \mathrm{~mL}$ of milk were taken from each sample. The babies included in the study were weight $>2500 \mathrm{~g}$. The Vitamin A concentration in the milk, before and after the processing, was found to be insufficient for that required $(46.57 \pm 6.28 \mu \mathrm{g} / \mathrm{dL}$ and $31.55 \pm 8.29 \mu \mathrm{g} / \mathrm{dL}$ respectively) for children who feed exclusively on HMB milk, notably premature babies, whose Vitamin A requirements are greater and whose capacity for ingesting milk is lower than that of fully born babies. The loss of Vitamin A through processing was, in this study, 32.51\%. To guarantee hepatic reserves and protect against deficiencies, it would be necessary that the vitamin A concentration in the milk be higher than $80 \mu \mathrm{g} / \mathrm{dL}$.

KEYWORDS: Vitamin A, retinol, maternal milk, Human Milk Bank.

1 Universidade Paulista. E-mail: anamaria67@vivax.com.br

${ }^{2}$ Instituto Nacional de Pesquisas da Amazônia. E-mail: marinho@inpa.gov.br 


\section{INTRODUÇÃO}

A vitamina $\mathrm{A}$, também conhecida como antiinfecciosa ou retinol é de vital importância para a saúde humana e de várias espécies animais. Está envolvida em processos fisiológicos como crescimento, na diferenciação celular, reprodução, sistema imunológico e no funcionamento e manutenção normal dos epitélios (WHO, 2002).

A deficiência em vitamina A (DVA) é um dos maiores problemas de nutrição em saúde pública em muitos países, afetando cerca de 190 milhóes de indivíduos no mundo. Em nível mundial, a DVA é o segundo problema nutricional mais prevalente (WHO, 2005).

Estudos realizados na década de 70 mostraram a prevalência considerável de sinais clínicos indicativos de DVA na populaçáo rural e urbana do Amazonas (Giugliano et al., 1978). A baixa ingestão dessa vitamina também foi verificada em vários inquéritos dietéticos como o nutriente mais limitante na dieta da população de Manaus (Shrimpton et al., 1984). Estudos mais recentes, também encontraram baixo consumo dietético e níveis séricos deficientes de vitamina A (Marinho et al., 1991; Marinho et al., 2003; Marinho \& Roncada, 2003).

Atualmente, uma das metas mais importantes no novo milênio é reduzir pela metade a taxa de mortalidade e morbidade infantil. Segundo o Unicef (2005), o aleitamento materno pode salvar a vida de 1,3 milhóes de crianças no mundo a cada ano, e também proteger sua saúde e crescimento.

O incentivo à amamentação é uma das estratégias que integram o Pacto Nacional pela Redução da Mortalidade Materna e Neonatal. Isso se deve à comprovaçáo dos benefícios do aleitamento para mães e bebês, reduzindo a incidência de câncer de mama e ovário nas mulheres, favorecendo o crescimento saudável do bebê e diminuindo a vulnerabilidade a várias doenças (Salviano, 2004).

Bancos de Leite Humano (BLH) são centros especializados de apoio, incentivo e promoção à amamentação, coleta e doação de leite de mulheres saudáveis, após processamento e controle de qualidade. O Brasil possui a maior rede de BLH do mundo, a qual se destaca, como excelente estratégia de política governamental e nutricional para alimentação saudável de recém-nascidos pré-termos de baixo peso, lactentes com doenças infecciosas, diarréicas e imunodeprimidas (Almeida et al., 1993), quando o leite de suas mães não é disponível. (Ribeiro et al., 2005). Assim, o aleitamento materno por meio do (BLH) constitui a mais sensível, econômica e eficaz intervenção para redução da morbimortalidade infantil.

Entretanto, alguns estudos têm mostrado que o processamento do leite no $\mathrm{BLH}$ pode alterar a sua composição com perdas de nutrientes sensíveis à açấo do oxigênio, de raios ultravioleta e temperatura elevada (Penteado, 2003). Sabe-se que a vitamina $\mathrm{A}$ é foto sensível e passível à açáo do oxigênio e relativamente estável ao calor (Roncada, 2003).

$\mathrm{Na}$ literatura poucos estudos abordam o manejo do leite humano distribuído pelo BLH, referente ao efeito do processamento (pasteurização) sobre os micronutrientes como a vitamina A. Assim, a qualidade do leite do BLH distribuído às crianças pré-termo que se alimentam exclusivamente com leite humano pode ser deficiente nessa vitamina, pois o catabolismo é muito elevado nas primeiras semanas após o nascimento e a necessidade nutricional de retinol é maior do que em qualquer outro estágio de vida (Ruffler et al., 2000; Dimenstein et al., 2003).

Considerando a relevância da alimentação infantil, o presente estudo tem por objetivo verificar o efeito do processamento do leite humano referente à vitamina $\mathrm{A}$, no BLH do Estado do Amazonas.

\section{MATERIAL E MÉTODOS}

\section{POPULAÇÃO}

Fizeram parte do estudo 50 máes doadoras cadastradas no BLH localizado na maternidade Ana Braga zona leste de Manaus/AM. A seleçáo para compor a amostra ocorreu conforme demanda espontânea. Para caracterização das mães, foram coletadas informaçôes através de formulários estruturados com questôes referentes às avaliaçôes cultural, sócio-econômica, sanitária, história patológica, dados do recém-nascido, dados antropométricos da doadora e questionário de freqüência alimentar e de fontes de vitamina A. Obteve-se o consentimento livre e esclarecido assinado por todas as participantes do estudo aprovado pelo Comitê de Ética do Instituto Nacional de Pesquisas da Amazônia (processo $n^{\circ}$ 026/2005).

\section{AVALIAÇÃO ANTROPOMÉTRICA}

Para obtenção do peso e altura das mães utilizou-se balança eletrônica e estadiômetro, respectivamente. Descalças e com roupas leves as participantes foram posicionadas em pé, no centro da base da balança e para a altura com os calcanhares juntos, em pé, costas retas e os braços estendidos ao lado do corpo, de acordo com as recomendaçôes de Jelliffe (1968). O Índice de Massa Corpórea (IMC) foi calculado e classificado de acordo com os seguintes pontos de corte: $>18,5 \mathrm{~kg} / \mathrm{m}^{2}$ (Baixo do peso); 18,5 a 24,9 Kg/m ${ }^{2}$ (Peso adequado); 25,0 a $29,9 \mathrm{Kg} / \mathrm{m}^{2}$ (Sobrepeso) (WHO, 1997).

\section{AVALIAÇÃO DIETÉTICA}

Foi usado um questionário de freqüência alimentar e de consumo de algumas fontes de vitamina A e carotenóides (IVACG, 1989). Foram obtidas, também as proporçōes de 
freqüências de outros alimentos por grupos, caracterizando o padrão alimentar das máes doadoras.

\section{ANÁLISE DE RETINOL E $\beta$-CAROTENO NO LEITE MATERNO ANTES} E APÓS PASTEURIZAÇÃO

O leite de cada doadora foi coletado em condiçóes assépticas, em frasco de vidro estéril e acondicionado no congelador doméstico, até ser enviado, semanalmente para o BLH. As amostras permaneceram congeladas até a pasteurização, antes, porém foram descongeladas lentamente em temperatura de $\pm 7^{\circ} \mathrm{C}$ em refrigerador e submetidas a uma temperatura de $40^{\circ} \mathrm{C}$ em banho-maria durante 10 minutos, fazendo-se homogeneizaçáo para uniformizar a temperatura. Foram retiradas alíquotas de $5 \mathrm{~mL}$ aproximadamente de leite de cada amostra, para análises em triplicata de retinol e ß-caroteno mediante a técnica de Cromatografia Liquida de Alta Eficiência (HPLC), segundo Arnaud et al. (1991).

A pasteurização foi realizada sob as seguintes condições: os frascos foram colocados em banho-maria a $65^{\circ} \mathrm{C}$, com uniformizador de temperatura durante 5 minutos, a partir deste tempo, o leite foi pasteurizado durante 30 minutos, até alcançar uma temperatura de $62,5^{\circ} \mathrm{C}$ na zona $\mathrm{D}$ do recipiente. As amostras pasteurizadas foram resfriadas em banho de gelo por 10 minutos e, em seguida retirado alíquotas, seguindo o mesmo procedimento para o leite náo pasteurizado. Em todas as etapas do processamento as amostras foram devidamente protegidas com papel alumínio com a finalidade de minimizar a degradação da vitamina A pela ação da luz.

Para os cálculos de consumo de vitamina A foi adotado as necessidades do lactente a termo: $400 \mu \mathrm{g} / \mathrm{dia}$, e do pré-termo $420 \mu \mathrm{g} / \mathrm{dia}$, conforme recomendaçóes da OIM estabelecidas como parâmetros, considerando um consumo de $500 \mathrm{ml}$ de leite/dia.

Para os cálculos do consumo dietético adotou-se as referencias diárias recomendadas para vitamina $\mathrm{A}$, de 400 $\mu \mathrm{g}$ para os lactentes a termo e $420 \mu \mathrm{g}$ para as pré-termo (DRI,2001)..

\section{ANÁLISE ESTATÍSTICA}

$\mathrm{Na}$ analise estatística foram aplicadas médias, medianas, desvio-padrão, comparação de médias através do teste t de Student.

\section{RESULTADOS E DISCUSSÃO}

As 50 mães avaliadas apresentaram idade entre de 17 e 37 anos, com peso médio de 63,16 $\pm 11,56 \mathrm{Kg}$ (Mínimo $=43,9$ e Máximo $=94,1)$, altura de $1,59 \pm 0,065 \mathrm{~cm}($ Min. $=1,52$ e Max. = 1,76). Observou-se que $50 \%$ das doadoras estavam com peso adequado. Entretanto, $28 \%$ das mães apresentaram sobrepeso. Sabe-se que o ato de amamentar contribui para recuperação rápida do peso adequado (Rea, 2004).

\section{CARACTERIZAÇÃO DOS RECÉM-NASCIDOS DAS MÃES DOADORAS}

Foi verificado que os 50 recém-nascidos apresentaram peso adequado ao nascer, ou seja, $>2,500 \mathrm{~g}$, conforme o ponto de corte proposto pela WHO (1980), com exceção de uma criança pré-termo. O peso médio total foi de 3,302 $\pm 747,62$ $\mathrm{kg}$, sendo $3,534 \pm 475,56 \mathrm{~kg}$ para as crianças do sexo masculino e 3,248 $\pm 673,33 \mathrm{~kg}$ para o feminino.

Segundo informações das doadoras do BLH/AM, foi destacado o tipo de parto cesáreo (60\%) seguido de (40\%) de parto normal (Tabela 1). A prática do parto cesariano foi alta no presente estudo contrariando a recomendaçáo da OMS de que os partos cesarianos náo devem ultrapassar 15\% do total de nascimentos (ENSP, 2009). Dados do Ministério da Saúde, em 2001, mostram que dos três milhóes de nascidos vivos no Brasil, aproximadamente, $38,1 \%$ nasceram de parto cesariano, sendo as menores proporçóes registradas nas regióes norte e nordeste ( $27,5 \%$ e $26,3 \%$, respectivamente). Esses dados náo conferem com os nossos resultados, sugerindo uma subnotificação dos tipos de parto na região norte. Em relação ao estado de saúde das doadoras do BLH/AM cerca de $86 \%$ das mães informaram que não apresentavam nenhuma patologia. Entretanto, 14\% delas apresentaram rinite, asma e anemia, patologias que parecem não comprometer a composição do leite. Quanto ao estado civil das doadoras do BLH/AM, $74 \%$ informaram que tem uniáo estável, sugerindo ser um fator positivo no estado emocional da mãe, contribuindo

Tabela 1 - Avaliação socioeconômicas das mães doadoras do BLH. Manaus/ AM, 2006.

\begin{tabular}{rc}
\hline Tipos de Parto & Cesáreo $60 \%$ \\
& Normal $40 \%$ \\
\hline Estado de Saúde & Saudável $86 \%$ \\
& Patologia $14 \%$ \\
Estado Civil & Casada $74 \%$ \\
& Solteira $26 \%$ \\
\hline Tipos de Moradia & Própria $62 \%$ \\
\hline Escolaridade & Ensino Médio Completo \\
\hline
\end{tabular}

para uma boa produçáo de leite. Nesse estudo $62 \%$ das mães doadoras possuíam casa própria, proporcionando estabilidade e segurança para as mesmas. Todas as mães eram alfabetizadas, sendo que $66 \%$ possuíam ensino médio completo, provavelmente um facilitador para obtençáo de conhecimento sobre a importância de amamentar e doar o excedente. Convém ressaltar que todas as máes receberam orientaçóes sobre técnicas de amamentação, demonstrando ser uma população consciente da importância do aleitamento materno para o bom desenvolvimento da criança. Vitolo et al (1999), não identificou a situação sócio econômica e escolaridade materna, em Campinas/SP como um fator 
significativo para a (DVA), embora a ingestão média dessa vitamina tenha sido inferior à de paises desenvolvidos.

\section{AVALIAÇÃO DIETÉTICA}

A Amazônia possui uma reserva nativa com muitas frutas de elevados teores de pró-vitamina A, tais como a pupunha e o

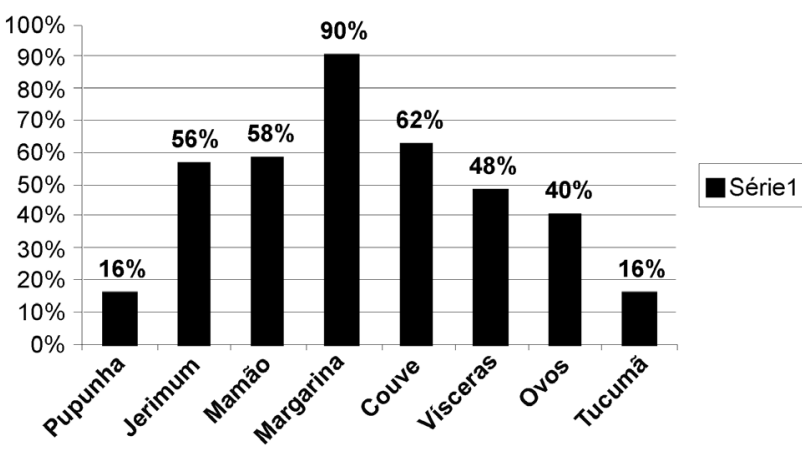

Figura 1 - Freqüência do Consumo de Alimentos Fontes de Vitamina A de Mães doadoras do BLH. 2006.

tucumã, entretanto, verificou-se que o consumo dessas frutas pela população estudada foi muito baixo, com percentuais de apenas 16\% (Figura 1). Dados da WHO (2005) indicam que nos países da América, aproximadamente 64\% da vitamina A ingerida é originada dos carotenóides, justificandose a necessidade do incentivo ao consumo de alimentos ricos em pró-vitamina $A$, às populaçôes que apresentam deficiência dessa vitamina. A margarina e as vísceras são também excelentes fontes de retinol e destacaram-se pela alta freqüência de consumo, $90 \%$ e $48 \%$ respectivamente, porem em pequenas quantidades. Segundo Underwood, (1994), a concentração de vitamina A no leite materno varia de acordo com a dieta da mãe.

\section{RETINOL E ß-CAROTENO NO LEITE MATERNO ANTES E APÓS PASTEURIZAÇÃO}

No presente estudo as concentraçóes de vitamina A encontradas, nos leites antes e após a pasteurização foram de 46,75 $\pm 6,28 \mu \mathrm{g} / \mathrm{ER} / \mathrm{dL}$ e de $31,55 \pm 8,29 \mu \mathrm{g} / \mathrm{ER} / \mathrm{dL}$, respectivamente, correspondendo a uma redução equivalente a $32,51 \%$. Esses resultados mostram que essas concentraçóes de retinol não são suficientes para suprir as necessidades de crianças que se alimentam exclusivamente do leite do BLH, notadamente as pré-termo, cujas necessidades de vitamina $\mathrm{A}$ são maiores e a capacidade de ingerir leite é menor que dos recém-nascidos a termo (Ribeiro et al., 2005). Assim, para garantir a formação de reservas hepáticas e a proteção contra deficiência, seriam necessárias concentraçóes de vitamina A no leite superiores a $80 \mu \mathrm{g} / \mathrm{dL}$. Ribeiro et al. (2005), no Rio Grande do Norte, também verificaram uma perda com o processamento do leite (pasteurização) de $34 \%$, sendo que antes do tratamento a concentração de retinol foi de $55,4 \pm$ $34,0 \mu \mathrm{g} / \mathrm{dL}$.
Alguns nutrientes podem variar de maneira significativa ao longo da lactação, durante o dia e até mesmo durante o período da mesma mamada, embora a composição do leite seja relativamente constante. Nesse contexto, a vitamina A destaca-se como um desses nutrientes que sofrem a influência do momento da mamada e do estágio de lactaçáo (Macias \& Schuweigert, 2001).

\section{COMPARAÇÃO DA PERDA DE RETINOL APÓS A PASTEURIZAÇÃO}

Comparando as concentraçóes de retinol lácteo, observa-se que nos leites crus e pasteurizados do presente trabalho (Tabela 2) são menores quando comparados aos estudos encontrados nos estados do Rio Grande do Norte e Rio de Janeiro, que apresentaram perdas consideráveis após pasteurização (Góes et al., 2002; Ribeiro, 2005). Entretanto a perda após a pasteurização, em nosso estudo foi menor, indicando que, provavelmente, houve um maior cuidado com as amostras durante o processamento. $\mathrm{O}$ descongelamento, a pasteurizaçáo e o congelamento são procedimentos que podem afetar o teor de diversos nutrientes, dentre eles a vitamina $A$.

Tabela 2 - Comparação da Concentração de Retinol no Leite Materno antes e após Pasteurização, segundo Autores. Manaus/AM, 2006.

\begin{tabular}{lccc}
\hline Leite Materno & \multicolumn{3}{c}{ Pasteurização } \\
\hline Estudos & Antes & Após & Perda (\%) \\
$\begin{array}{l}\text { Ribeiro et al, } \\
2005 \text { (RN) }\end{array}$ & $55,40 \mu \mathrm{g}$ & $36,60 \mu \mathrm{g}$ & 34,00 \\
$\begin{array}{l}\text { Góes, 2002 (RJ) } \\
\text { Nossos }\end{array}$ & $51,74 \mu \mathrm{g}$ & $31,46 \mu \mathrm{g}$ & 39,19 \\
$\begin{array}{l}\text { Resultados. } \\
\text { 2005/2006 }\end{array}$ & $46,75 \mu \mathrm{g}$ & $31,55 \mu \mathrm{g}$ & 32,51 \\
\hline
\end{tabular}

RECOMENDAÇÃO DA INGESTÃO DE VITAMINA A SEGUNDO A (DRl'S)

De acordo com as Dietary Reference Intakes (DRI's), a ingestão recomendada de vitamina A para crianças de zero a seis meses de idade é de $400 \mu g$ por dia e para os recém-nascidos pré-termo é de $420 \mu \mathrm{g} / \mathrm{dia}$ segundo a (DRI's) (IOM, 2000).

Com a finalidade de estimar o consumo diário de vitamina A, considerou-se um aporte de $500 \mathrm{~mL}$ para crianças < de um mês e de $750 \mathrm{~mL}$ de leite humano para aqueles de 1 a 6 meses de idade. Como pode se observar a Tabela 3, as percentagens de adequação de vitamina A no leite materno cru (LHOC) e pasteurizado (LHOP), em função das recomendaçôes dietéticas. Assim, o (LHOC) cobriria 58,40\% dos requerimentos de vitamina A e o (LHOP), apenas 39,40\% do aporte diário recomendado para o recém-nascido a termo, com menos de um mês de vida. Para os recém-nascidos prematuros os teores de vitamina A (LHOC e LHOP) situaram-se muito abaixo das recomendaçóes $(55,60 \%$ e $37,55 \%)$, respectivamente. Com relação a faixa etária de crianças de 1 a 6 meses de vida, 
a contribuição de retinol no leite também não cobriu as recomendaçôes dietéticas, entretanto, foi melhor do que para crianças menores de um mês de idade, ou seja acima de 55\% de adequação tanto para o recém-nascido a termo e pré-termo.

Tabela 3 - Ingestão Recomendada de Vitamina A nos leites crus e pasteurizados segundo as necessidades diárias dos recém-nascidos a termo e prematuros. Manaus/AM 2006.

\begin{tabular}{|c|c|c|c|c|c|c|}
\hline $\begin{array}{l}\text { Idade } \\
(0 \text { a } 6\end{array}$ & Recé & m-nasci & 0 a termo & Recér & 1-nascidc & prematuro \\
\hline $\begin{array}{l}\text { DRl'S, } \\
2001 . \\
\text { Vitamina A }\end{array}$ & $400 \mu \mathrm{g}$ & $\begin{array}{l}\text { Leite } \\
\text { Cru }\end{array}$ & $\begin{array}{c}\text { Leite } \\
\text { Pasteurizado }\end{array}$ & $420 \mu \mathrm{g}$ & $\begin{array}{c}\text { Leite } \\
\text { Cru }\end{array}$ & $\begin{array}{c}\text { Leite } \\
\text { Pasteurizado }\end{array}$ \\
\hline $\begin{array}{l}\text { Consumo } \\
\text { médio } \\
\text { (<30 dias) }\end{array}$ & $500 \mathrm{ml}$ & $58,40 \%$ & $39,40 \%$ & $500 \mathrm{ml}$ & $55,60 \%$ & $37,55 \%$ \\
\hline $\begin{array}{l}\text { Consumo } \\
\text { médio } \\
\text { (1 a } 6 \\
\text { meses) }\end{array}$ & $750 \mathrm{ml}$ & $87,60 \%$ & $59,10 \%$ & $750 \mathrm{ml}$ & $83,48 \%$ & $56,33 \%$ \\
\hline
\end{tabular}

No (LHOC) variou de $83,48 \%$ a $87,60 \%$ e no LHOP variou de $56,33 \%$ 59,10\%.

Nesse contexto, o estado nutricional do recém nascido está intimamente ligado ao aporte do leite materno durante as primeiras semanas de sua vida, desse modo, a produção de leite náo deve ser considerada somente do ponto de vista quantitativo, mas também a sua qualidade, para qual o conhecimento da concentraçáo de micronutrientes principalmente a vitamina A é de suma importância, podendo servir como referencia para estabelecer os requerimentos mínimos nutricionais do recém nascido.

\section{CONCLUSÕES}

As concentraçóes de vitamina A no leite antes e após o processamento, não são suficientes para suprir as necessidades nutricionais diárias de lactentes que se alimentam exclusivamente do leite do $\mathrm{BLH}$, notadamente as pré-termo, cujos requerimentos de vitamina A são maiores. Sugere-se a promoção ao estímulo do consumo de alimentos de origem animal e vegetal ricos em vitamina $\mathrm{A}$, pelas máes doadoras, principalmente durante o período de lactação visando aumentar a concentração deste nutriente no leite.

\section{BIBLIOGRAFIA CITADA}

Almeida, J.A.G.; Novak, F.R.; Sandoval, M. H. 1993. Recomendaçóes técnicas para los bancos de leche humana II. Control de calidad. Puericultura y Pediatria, 61: 12-15.

Arnaud, J.; Fortis, S.; Blachier, D.K.I.A., Favier, A. 1991. Simultaneous determination of retinol, $\alpha$-tocopherol and Betacarotene in serum by isocratic high-performance liquid cromatography. Journal of Chromatography, 572: 103-116.
ENSP.2009. Disponível em (http://www.ensp.fiocruz.br) Acesso: $17 / 08 / 2009$

Dimenstein, R.; Simplicio, J.L.; Ribeiro, K.D.; Melo, I.L. 2003. Influência de variáveis socioeconômicas e de saúde maternoinfantil sobre os níveis de retinol no colostro humano. Jornal de Pediatria, 89: 513-518.

Giugliano, R.; Albuquerque, H.C.R.; Shrimpton, R. 1978. Estudo antropométrico e clínico e de padróes alimentares em um grupo de escolares de Manaus. Acta Amazonica, 8: 75-82.

Góes, H.C.; Torres, A.G.; Donange C.M.; Trugo, N.M. 2002. Nutrient composition of blanked human milk in Brazil and influence of processing on zinc distribution in milk fractions. Nutrition, 18: 590-594.

International Vitamin A Consultative Group (ICACG). 1989. Guidelines for the for development of a simplified dietary assessment to identify groups at risk for inadequate intake of vitamin A: report of international vitamin A. Washington. IVACG, p. 1-18.

Institute of Medicine (IOM). 2000. Dietary References Intakes for Vitamin A, Vitamin K, Arsenic, Borom, Chromium, Copper, Iodine, Iron, Menganese, Molybdenium, Nickel, Silicon, Vanadium and Zinc. Washington, DC, National Academic Press. IOM.

Jelliffe, D.B. 1968. The assessment of the comunit. Geneve. Who. $\mathrm{N}^{\circ} 53$.

Macias, C.; Schuweigert, F.J. 2001. Changes in the concentration of carotenoid, vitamin A, alpha-tocopherol and total lipids in human milk throughout early lactation. Annals Nutritional Metabolism, 45: 82-85.

Marinho, H.A.; Shrimpton, R.; Giugliano, R.; Burini, R.C. 1991. Influence of enteral parasites on the blood vitamin levels in preschool children orally supplemented with retinol and / or zinc. European Journal Clinic Nutritional, 45: 539-44.

Marinho, H.A.; Roncada, M.J. 2003. Ingestão e hábitos alimentares de pré-escolares de três capitais da Amazônia Ocidental Brasileira: um enfoque especial à ingestão de vitamina A. Acta Amazonica, 33: 263-274.

Marinho, H.A.; Castro J.S.; Alencar, F.H.; Yuyama, L.; Donadio, M.; Batista, V.L. 2003. Deficiência subclínica de vitamina A em crianças de 3 a 12 anos de idade da periferia da cidade de Manaus/AM/Brasil. Revista do Hospital Universitário Getúlio Vargas, 2: 36-44.

Penteado, M.D. 2003. Vitaminas: aspectos nutricionais, bioquimicos, clinicos e analiticos. Disseratção de Mestrado, Barueri, São Paulo, $74 \mathrm{pp}$.

Rea, M.F. 2004. Os benefícios da amamentação para a saúde da mulher. Jornal de Pediatria, p.142-146.

Ribeiro, K.D.S.; Melo, I.L.P.; Pristo, A.Z.O.; Dimenstein, R. 2005. Efeito do processamento do leite humano sobre os níveis de retinol. Jornal de Pedriatria, 79: 513-518.

Roncada, M.J. 2003. Vitamina A, p. 167-178. In: Dutra de Oliveira, J. E \& Machine, J. S. Ciências Nutricionais. São Paulo. Sarvier.

Ruffler, C.P.; Ruffler, J.G.; Pagani, J.R.; Barreto, A.P.; Braz, M.G. 2000. Neonatologia, p. 1051-1086. In: Waltzberg, D. L. Nutrição oral, enteral e parenteral na prática clínica. Atheneu, São Paulo. 
Shrimpton, R.; Marinho, H.A.; Giugliano, R.; Giugliano L.G. 1984. Estudos nutricionais das populaçóes rurais da Amazônia. II. Rio Negro. Acta Amazonica, 14: 427-49.

Salviano, S. 2004. Importância do hábito de amamentar. Revista de Nutrição, 67: 8 .

Underwood, B.A. 1994. Maternal vitamin A status and its importance in infancy and early childhood. American Journal of Clinical Nutrition, 59: 517S-24S.

Unicef, 2005. Aleitamento materno. Disponível em (http://www. unicef.org.) Acesso 17/03/2006.

Vitolo, M.R; Accioly,E; Ramalho, R.A; Cardoso, C.B; Carvalho, E.B. 1999. Níveis de vitamina A no leite maduro de nutrizes adolescentes e adultas de diferentes estratos sócio-economicos. Revista Ciências Médicas, 8: 3-10.
Word Health Organization (WHO), 1980. The incidence of low birthweight: a critical review of available information. World Health Statistics Quarterly, Word Health Organization (WHO). 2005 33: 197-244.

Word Health Organization (WHO). 1997. Physical status: the use and interpretation of anthropometry. Geneva. Technical reports series, n. 854 .

Word Health Organization (WHO). 2002. The optima duration of exclusive breastfeeding. Results of a who systematic review. $36 \mathrm{pp}$.

Word Health Organization (WHO). 2005. Global prevalence of vitamin A deficiency. Geneva.

Recebido em: 27/11/2007

Aceito em: 13/10/2009 